\title{
Ultrasound-guided arthroscopic management of hallux rigidus
}

\author{
Łukasz M. Paczesny ${ }^{1}$, Jacek Kruczyński ${ }^{2}$ \\ ${ }^{1}$ Orvit Clinic, Citomed Health Centre, Torun, Poland \\ ${ }^{2}$ Department of Orthopaedics, Orthopaedic Oncology and Traumatology, Poznan University of Medical Science, Poznan, Poland \\ Videosurgery Miniinv 2016; 11 (3): 144-148 \\ DOI: $10.5114 /$ wiitm.2016.62811
}

\begin{abstract}
Introduction: The use of metatarso-phalangeal joint arthroscopy in the treatment of osteochondritis dissecans was first described in 1988. The technique produces good results. However, it can be difficult to enter a joint when it is deformed by degenerative disease. Sonography is a modern visualisation modality which can be used in orthopaedic surgery.

Aim: To describe a method of intraoperative sonographic navigation during first metatarso-phalangeal joint arthroscopy.

Material and methods: The modality was used in 3 patients. The joint was visualised in the ultrasound scanner. After confirming the intra-articular position of the guide needle, a medial portal was established. The procedure started with the removal and vaporisation of the hypertrophic synovium. Gradual resection of the osteophytes was then carried out. The procedure was terminated after the ultrasound image showed that a smooth upper surface of the metatarsal head had been achieved.

Results: All 3 patients were satisfied with the procedure and function of the treated feet. Average surgery time was $81 \mathrm{~min}$. No complications were found.

Conclusions: Mini-invasive treatment of hallux rigidus with sonography-guided arthroscopic cheilectomy appears to be a reproducible procedure leading to good clinical results. We encourage surgeons familiar with ultrasound visualisation of the joints to use the technique described in this paper in the arthroscopic treatment of hallux rigidus.
\end{abstract}

Key words: arthroscopy, ultrasound-guided, ultrasonography, hallux rigidus.

\section{Introduction}

The use of metatarso-phalangeal joint arthroscopy in the treatment of osteochondritis dissecans was first described by Bartlett in 1988 [1]. Five years later van Dijk published his paper on arthroscopic cheilectomy of hallux rigidus [2, 3]. The technique presented by these researchers produces good results. Some papers describe the use of traction to improve joint space visualisation [4]. However, it can be difficult to enter a joint when it is deformed by degenerative disease. Visualisation of the dorsal surface of the metatarsal head can be challenging, especially if it is deformed by osteophytes. In many hallux rigidus cases the joint cavity is filled with hypertrophic synovium, making the initial visualisation of the upper surface of the metatarsal head difficult. Sonography is a modern visualisation modality which can be used in orthopaedic surgery. It is widely used, not only as an aid to diagnosis but also during the surgical procedures themselves. In 2007 de Lucia discussed the simultaneous use of arthroscopy and ultrasound in the surgical treatment of rheumatoid arthritis. In these cases sonography, with the use of power Doppler, allows intraoperative 
localisation of synovial hypertrophy [5]. Another advantage of intraoperative sonography is the possibility it provides of visualising vessels and nerves while establishing arthroscopic portals. This possibility is especially useful in hip arthroscopy, where injury to these structures may result in severe complications $[6,7]$, although such complications were also reported in knee procedures [8].

\section{Aim}

The aim of the study was to describe a method of intraoperative sonographic navigation during first metatarso-phalangeal joint arthroscopy. To prove that it allows a constant view of osteophyte removal, ensuring complete achievement of the procedure's goal.

\section{Material and methods}

The modality was used in three patients: two women and one man. To provide good ultrasound penetration through sterile draping, the transducer was covered with ultrasound gel before draping, and more gel was added frequently during the visualisation. After this sterile draping of the ultrasound transducer (linear probe $7.5 \mathrm{~cm}$, frequency 10-12 MHz) the joint was visualised in the ultrasound scanner by placing the probe longitudinally over the upper surface of the first metatarso-phalangeal joint (Photo 1$)$. Initially, an injection needle $(0.8 \mathrm{~mm})$ was introduced from the medial to the lateral aspect of the joint (Photo 2), producing a typical, hyperechoic dot-like echo (Photo 3). After confirming the intra-articular position of the needle under ultrasound guidance, the joint was injected with approximately $2 \mathrm{ml}$ of physiological saline ( $0.9 \%$ sodium chloride). The needle was then removed and a medial portal was established according to the previous needle position, still under ultrasound guidance (Photos 4 and 5). After insertion of the arthroscope $(4.0 \mathrm{~mm}$, Smith and Nephew, Photo 6) the joint cavity was visualised and carefully inspected (Photo 7). The procedure started with the removal and vaporisation of the hypertrophic synovium. Gradual resection of the osteophytes was then carried out according to the van Dijk technique [3], using a shaver (Photos 8 and 9). During this procedure an ultrasound scanner was used to check the surface of the metatarsal bone. The procedure was terminated after the ultrasound image showed that a smooth upper surface of the metatarsal head had been achieved (Photos 10 and 11).

\section{Results}

All 3 patients were satisfied with the procedure and function of the operated feet (Photo 12). The patients' data, including age, procedure time, follow-up time and changes in the range of motion are summarized in Table I. Both women returned to full

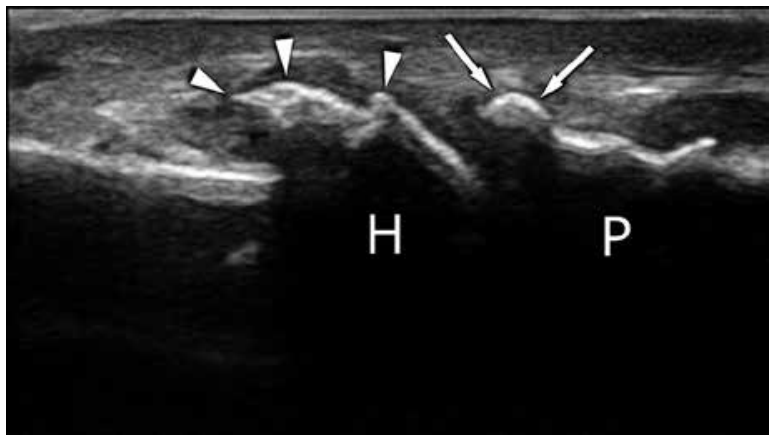

Photo 1. Preoperative sonography of patient no. 1

$H$ - first metatarsal head, $P$ - proximal phalanx of the toe, arrowheads - needle echo.

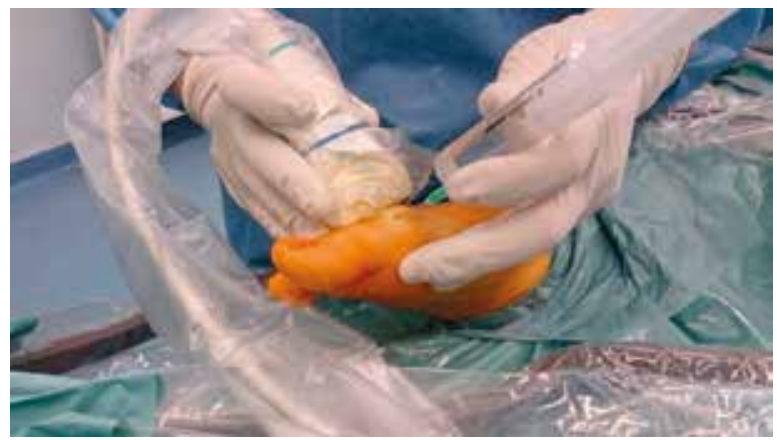

Photo 2. Ultrasound transducer placement during needle insertion under ultrasound guidance

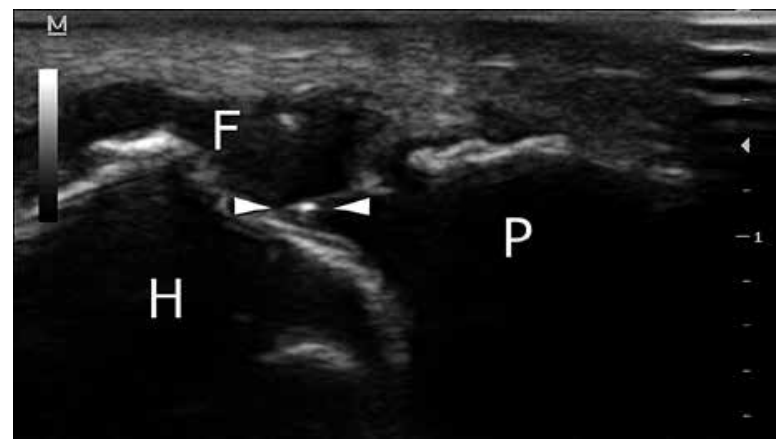

Photo 3. Sonography of the intra-articular needle position after injection of $2 \mathrm{ml}$ of physiological saline

$H$ - first metatarsal head, $P$-proximal phalanx of the toe, $F$-fluid injected into the joint, arrowheads - needle echo. 


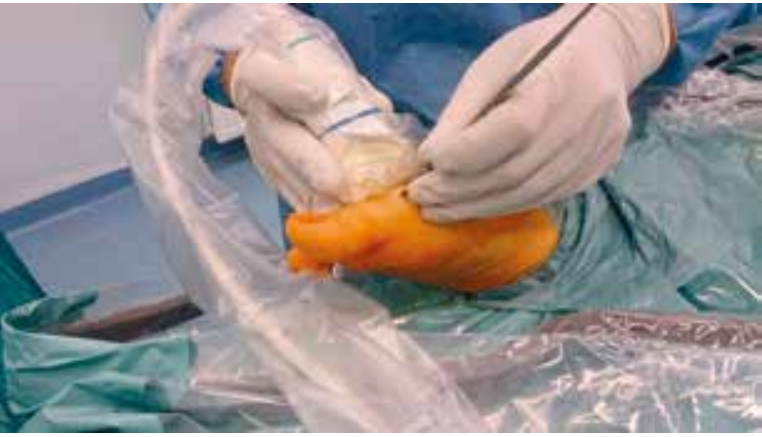

Photo 4. Medial portal established under ultrasound guidance

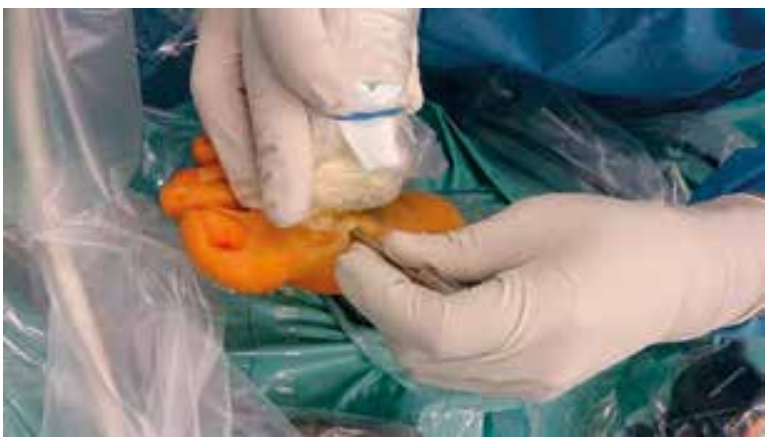

Photo 6. Arthroscope's sheath insertion

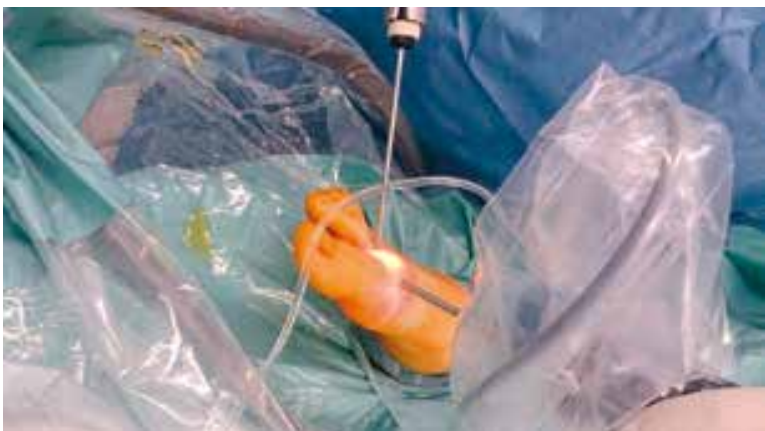

Photo 8. Arthroscopic resection of the osteophytes using a shaver

activity - the younger one is a professional dancer, and the older one returned to fitness activity 3 times a week. The male patient is a factory worker who was able to return to his previous job. No complications were found.

\section{Discussion}

The use of arthroscopy in first metatarsophalangeal joint treatment has been becoming

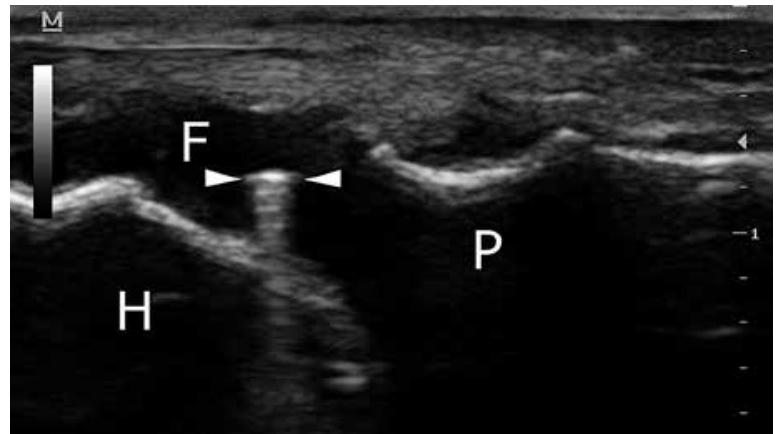

Photo 5. Sonography-guided arthroscopic portal placement

$H$ - first metatarsal head, $P$-proximal phalanx of the toe, $F$ - fluid injected into the joint, arrowheads - needle echo.

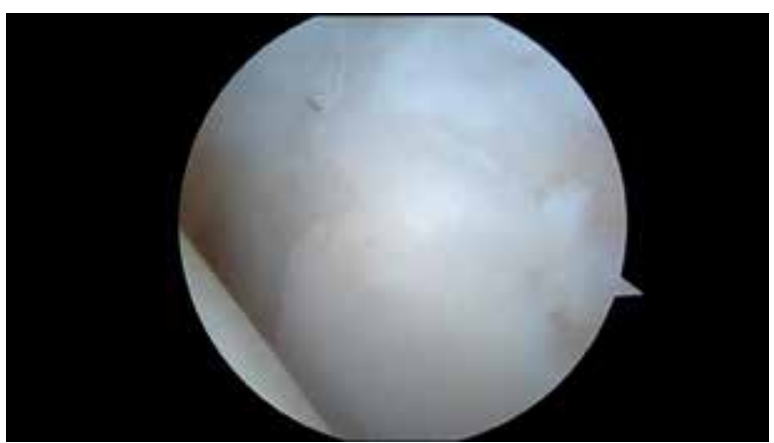

Photo 7. Arthroscopic view of the proximal phalanx articular surface and joint space

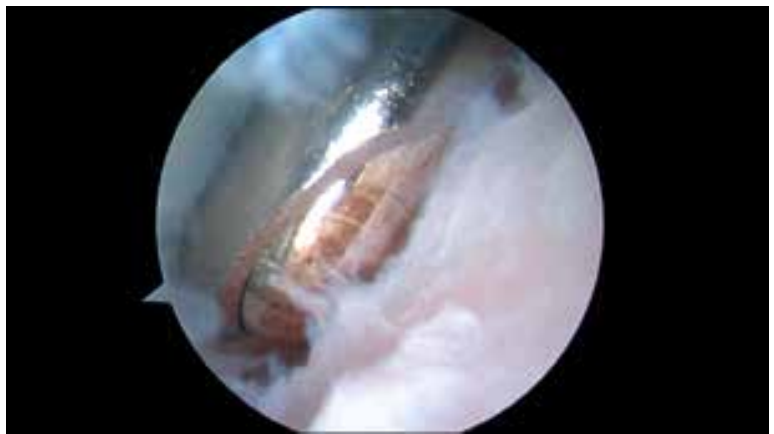

Photo 9. Intra-articular view of osteophyte resection

more popular in recent years. It has been used to treat osteochondritis dissecans, hallux valgus and gout, but the most common indication is hallux rigidus [9-11]. In his review of the relevant literature, Roukis stated that "it is clear that there is a low incidence of revision surgery required after isolated cheilectomy for hallux rigidus" [12]. Walter and Perera compared classic open cheilectomy with arthroscopic and fluoroscopy-assisted percutaneous techniques in the treatment of hallux rigi- 


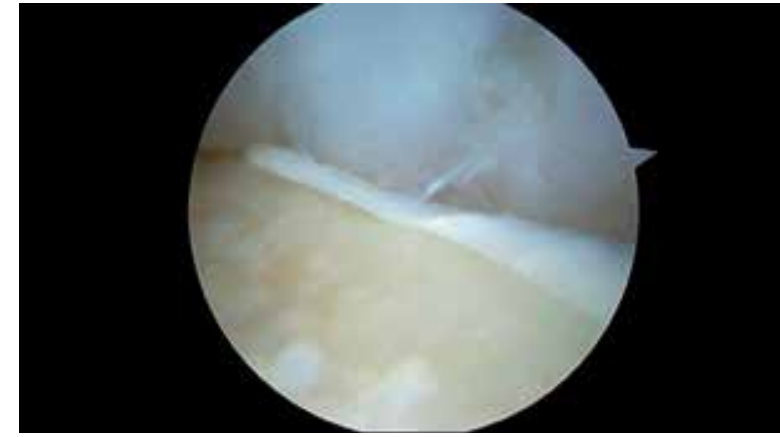

Photo 10. Upper margin of the first metatarsal articular surface

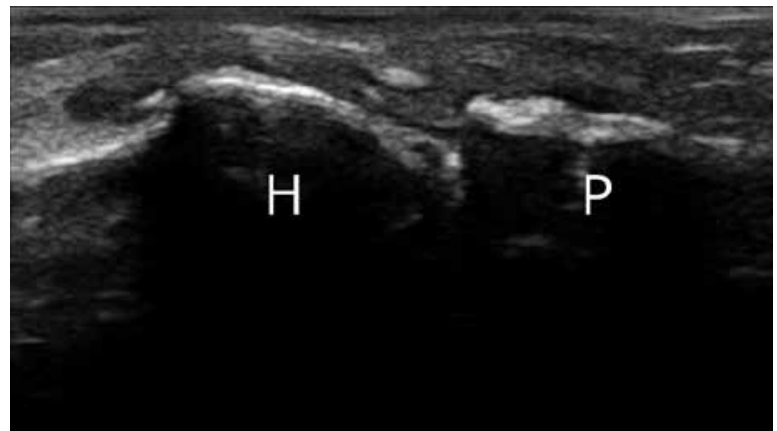

Photo 11. Sonography of the upper surface of the first metatarsophalangeal joint confirming complete removal of osteophytes

$H$ - first metatarsal head, $P$ - proximal phalanx of the toe
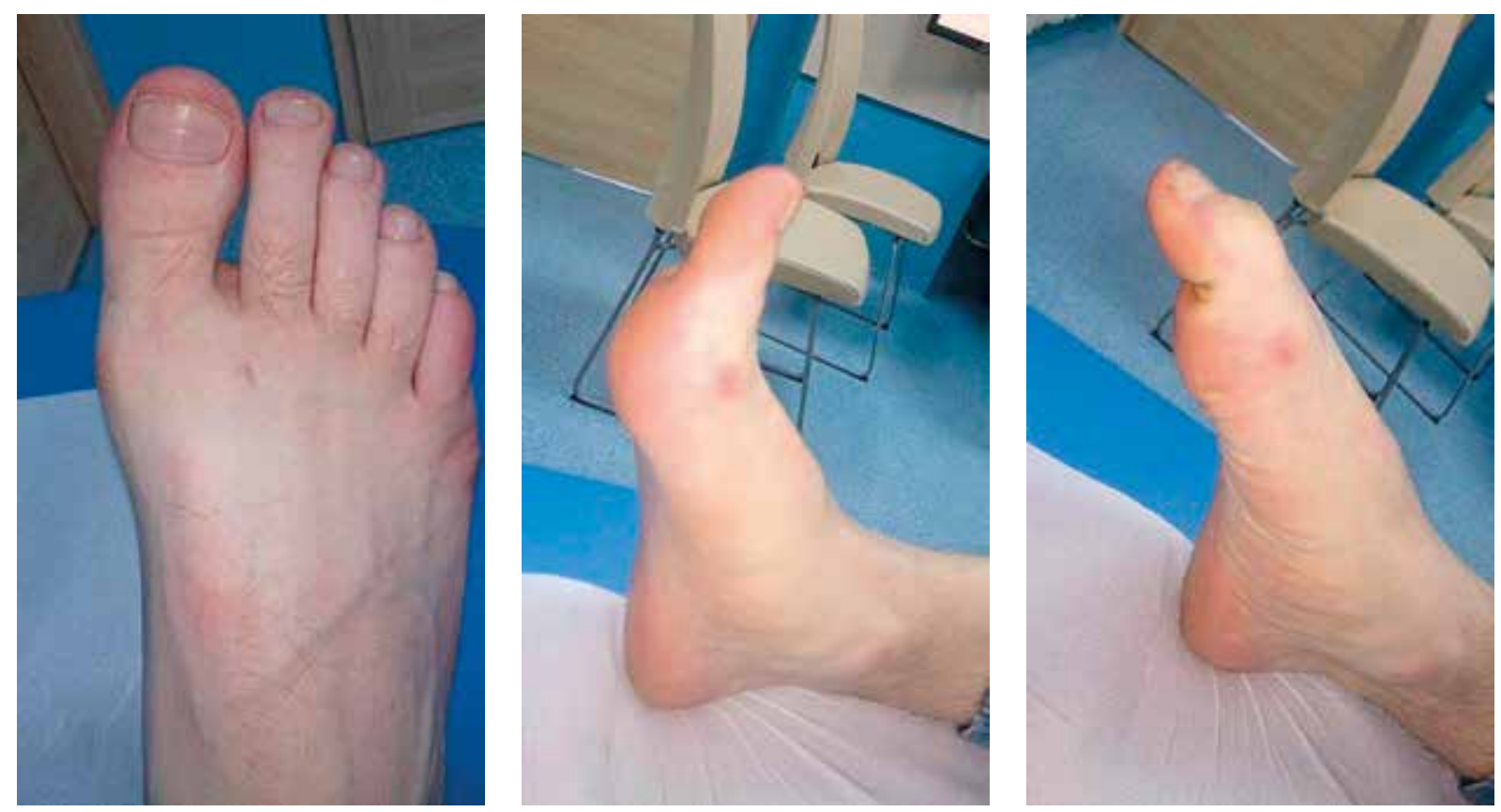

Photo 12. Patient no. 2. Outcome of the procedure 6 months postoperatively

Table I. Patient characteristics

\begin{tabular}{|lcccccccc|}
\hline $\begin{array}{l}\text { Patient } \\
\text { no. }\end{array}$ & Gender & $\begin{array}{c}\text { Age } \\
\text { [years] }\end{array}$ & $\begin{array}{c}\text { Procedure } \\
\text { duration }[\mathrm{min}]\end{array}$ & $\begin{array}{c}\text { Follow-up } \\
{[\text { months] }}\end{array}$ & $\begin{array}{c}\text { Preoperative } \\
\text { arc }\left[^{\circ}\right]\end{array}$ & $\begin{array}{c}\text { Preoperative } \\
\text { dorsiflexion }\left[{ }^{\circ}\right]\end{array}$ & $\begin{array}{c}\text { Postoperative } \\
\text { arc }\left[{ }^{\circ}\right]\end{array}$ & $\begin{array}{c}\text { Postoperative } \\
\text { dorsiflexion }\left[{ }^{\circ}\right]\end{array}$ \\
\hline 1 & F & 47 & 80 & 20 & 10 & 0 & 75 & 55 \\
\hline 2 & M & 38 & 95 & 9 & 25 & 10 & 75 & 60 \\
\hline 3 & F & 17 & 70 & 4 & 30 & 0 & 70 & 40 \\
\hline
\end{tabular}

F-female, $M-$ male.

dus. They concluded that these newer techniques "are encouraging, although large, well-designed comparative trials to identify a benefit over open cheilectomy have not yet been conducted" [13]. Ar- throscopic management of this condition provides good results and is less invasive than open cheilectomy. However, it can be challenging to establish portals without harming cartilage and to achieve 
an optimal position for the removal of osteophytes. Some authors have suggested the use of a c-arm to confirm the proper placement of the instruments in the joint [14]. The use of sonography described in our paper does not require c-arm involvement. During the procedure it can be difficult to ensure complete removal of any bony eminence without ultrasound guidance. Even with extended synovectomy the collapsing capsule and synovium can confuse the surgeon. The first metatarsal joint is relatively small, and navigation over the upper surface of the metatarsal head, under both the capsule and the extensor tendon, requires good orientation. Hunt recommended converting to the open technique if it is impossible to safely access and remove the osteophyte(s) due to capsular scarring of the osteophyte [15]. Thanks to ultrasound imaging the conversion to an open procedure can be omitted. Double visualisation of the deformation improves the surgeon's confidence and makes the procedure safer and more reliable. For surgeons familiar with joint ultrasound the use of this double visualisation modality will provide faster and more accurate portal establishment. This way the resection can be performed more accurately.

The weakness of this paper is the small group of patients treated with this double visualisation technique.

Our results in this pilot study were comparable to those presented by lqbal and Chana in 1998 [16]. The impact of this new double visualisation technique (arthroscopy plus sonography) on the longterm results should be investigated in a larger group.

The disadvantage of this modification is the need for extra equipment in the operating room and the additional cost of the needle, ultrasound gel and draping. It should also be noted that basic ultrasound skills are mandatory for such procedures. Both members of our team, the surgeon and his assistant, are experienced sonographers.

\section{Conclusions}

Mini-invasive treatment of hallux rigidus with sonography-guided arthroscopic cheilectomy appears to be a reproducible procedure leading to good clinical results. We encourage surgeons familiar with ultrasound visualisation of the joints to use the technique described in this paper in the arthroscopic treatment of hallux rigidus.

\section{Conflict of interest}

The authors declare no conflict of interest.

\section{References}

1. Bartlett DH. Arthroscopic management of osteochondritis dissecans of the first metatarsal head. Arthroscopy 1988; 4: 51-4.

2. van Dijk CN. Arthroscopy of the MTP-I joint. Acta Orthop Scand 1993; 64 (Suppl. 253): 11.

3. van Dijk CN, Veenstra KM, Nuesch BC. Arthroscopic surgery of the metatarsophalangeal first joint. Arthroscopy 1998; 14: 851-5.

4. Siclari A, Piras M. Hallux metatarsophalangeal arthroscopy: indications and techniques. Foot Ankle Clin 2015; 20: 109-22.

5. De Lucia O, Paresce E, Murgo A, et al. Simultaneous ultrasonography and arthroscopy for the study of the joint environment: indications and limits. Reumatismo 2007; 59: 146-52.

6. Hua Y, Yang Y, Chen S, et al. Ultrasound-guided establishment of hip arthroscopy portals. Arthroscopy 2009; 25: 1491-5.

7. Weinrauch P, Kermeci S. Ultrasound-assisted hip arthroscopy. Arthrosc Tech 2014; 3: e255-9.

8. Neagoe RM, Bancu S, Muresan M, Sala D. Major vascular injuries complicating knee arthroscopy. Videosurgery Miniinv 2015; 10: 266-74.

9. Lui TH. Arthroscopy and endoscopy of the foot and ankle: indications for new techniques. Arthroscopy 2007; 23: 889-902.

10. Lui TH. First metatarsophalangeal joint arthroscopy in patients with hallux valgus. Arthroscopy 2008; 24: 1122-9.

11. Lui TH. Endoscopic resection of the gouty tophi of the first metatarsophalangeal joint. Arch Orthop Trauma Surg 2008; 128: 521-3.

12. Roukis TS. The need for surgical revision after isolated cheilectomy for hallux rigidus: a systematic review. J Foot Ankle Surg 2010; 49: 465-70.

13. Walter R, Perera A. Open, arthroscopic, and percutaneous cheilectomy for hallux rigidus. Foot Ankle Clin 2015; 20: 421-31.

14. Schmid T, Younger A. First metatarsophalangeal joint degeneration: arthroscopic treatment. Foot Ankle Clin 2015; 20: 413-20.

15. Hunt KJ. Hallux metatarsophalangeal (MTP) joint arthroscopy for hallux rigidus. Foot Ankle Int 2015; 36: 113-9.

16. Iqbal MJ, Chana GS. Arthroscopic cheilectomy for hallux rigidus. Arthroscopy 1998; 14: 307-10.

Received: 1.05.2016, accepted: 15.09.2016. 